\title{
A review of seven support surfaces with emphasis on their protection of the spinally injured
}

\author{
P W Main, M E Lovell
}

\begin{abstract}
The aim was to evaluate seven evacuation support surfaces. These included the conventional spinal board, two designs of vacuum stretcher, a prototype support surface which was a combination of both principles, and three conventional stretchers. Interface pressures were evaluated in four healthy volunteers. The sacral and thoracic interface pressures were measured. Mean sacral readings were: spinal board $233.5 \mathrm{~mm} \mathrm{Hg}$, old vacuum stretcher $139 \mathrm{~mm} \mathrm{Hg}$, new design $94.8 \mathrm{~mm} \mathrm{Hg}$, prototype board 119.5 mm Hg, York Two stretcher $46 \mathrm{~mm} \mathrm{Hg}$, Army stretcher $61 \mathrm{~mm} \mathrm{Hg}$, and the PVC and aluminium stretcher $66 \mathrm{~mm} \mathrm{Hg}$. Thoracic pressure readings were: spinal board $82.9 \mathrm{~mm} \mathrm{Hg}$, old design vacuum stretcher $58 \mathrm{~mm} \mathrm{Hg}$, new design 37.8 $\mathrm{mm} \mathrm{Hg}$, prototype board $53.7 \mathrm{~mm} \mathrm{Hg}$, York two $21 \mathrm{~mm} \mathrm{Hg}$, army stretcher $35 \cdot 4$ $\mathrm{mm} \mathrm{Hg}$, and PVC stretcher $38.5 \mathrm{~mm} \mathrm{Hg}$. Analysis of variance showed both distributions to be highly significant $(P<0 \cdot 001)$. The spinal board has several deficiencies, including lack of support for the lumbar lordosis. It should not be the preferred surface for the transfer of patients with spinal injuries.

( f Accid Emerg Med 1996;13:34-37)
\end{abstract}

Key terms: pressure necrosis; spinal board; spinal injuries; vacuum stretcher.

The initial management and treatment of the spinal injured or multiply injured patient must include careful consideration of the collection and transport of the casualty. If the casualty is spinally injured it is important that neurological injury is not created or aggravated. It is also necessary for support surfaces to be easy to use, durable, and compatible with conventional vehicles. The support surface system should also permit $x$ rays to be taken without degradation of the image and should not impair access to the casualty to allow cervical spine control and intravenous cannulation.

Attention needs to be given to the amount of time that the casualty must spend on the support surface. Pressure sores caused by continued pressure over bony prominences have been strongly linked to long transport times of casualties on poor support surfaces and can cause major complications. ${ }^{1}$ It is not just the elderly or infirm who are at risk of pressure sores, but any patient who is acutely ill or shocked because under such conditions tissue perfusion will be compromised. ${ }^{2}$ One study suggested that $59 \%$ of spinally injured patients suffer from pressure sores caused in the acute injury phase, and the likelihood of pressure sores was also strongly linked with the time on the spinal board. ${ }^{1}$ Pressure sores can be a serious complication of spinal injury, causing a weakening of the ability of the casualty to combat his injury as well as delaying discharge from hospital. In the present cash conscious health service it is considered that pressure sores may be costing $£ 321$ million pounds per year to treat. ${ }^{3}$ Plastic surgery may be required to close the defect in the skin.

Pressure sores occur when the skin and deep tissues are deprived of their blood supply for any significant length of time, usually between two and six hours. The rate of pressure sore necrosis is inversely proportional to the level of pressure applied: in one experiment Daniel et al showed that $500 \mathrm{~mm} \mathrm{Hg}$ applied for two hours or $100 \mathrm{~mm} \mathrm{Hg}$ applied for 10 hours would cause muscle damage in pigs. ${ }^{4}$ The pressure that causes the damage is produced by the weight of the patient pressing the skin and tissues against the support surface. This squeezes out the blood and closes the blood vessels that enter these tissues. ${ }^{5}$ It is considered that blood flow will still occur to some degree to these vulnerable areas even if the pressure at the surfaces approaches systolic blood pressure. However, blood flow to these areas decreases if the interface pressures are greater than $30 \mathrm{~mm} \mathrm{Hg} .{ }^{6}$ Interface pressure is that pressure which can be recorded between two surfaces (the casualty and the support surface). Interesting information has been provided with the advent of pressure sensors and meters that can be inserted between the casualty and support surface to record the pressures.

Many different types of support surface are available. It is often thought that the flat spinal board is the best surface if spinal injury is suspected, although this has not been substantiated by any research. The spine is not flat and little support is given to some areas, most of the weight of the body being taken by the sacrum and thoracic kyphosis, with no support given to the lumbar lordosis (fig 1). An unstable injury caused by a spinal fracture at this level may cause collapse of the spinal curvature; this is not prevented by the spinal board, and neurological damage may occur. It is well established that the sacrum and thoracic areas have a high rate of pressure sore formation; this is because they are the only areas in contact with the spinal board. ${ }^{1}$

An alternative to the spinal board is the vacuum stretcher, a flexible sack of polystyrene beads that becomes rigid on the application of 


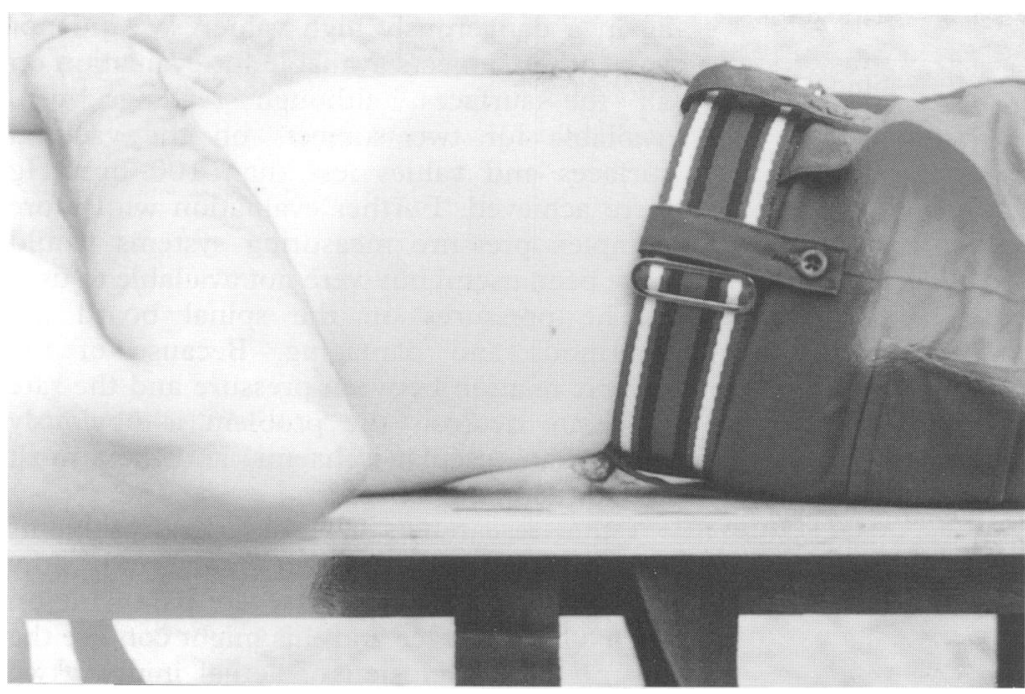

Fig 1 Subject on a spinal board. Failure of support of the lumbar lordosis is demonstrated.

a vacuum. These have in the past been used to treat limb injuries, acting as rigid splints. A larger version is available as a full stretcher and can provide superior support to the spine. The major disadvantage of the vacuum stretcher is its bulk: it is wider than most ambulance trolleys and therefore not compatible with some vehicles; it is also bulky to store when not in use. Efforts have been made recently to decrease the width of the vacuum stretcher to make it more acceptable to the ambulance service. One of our aims was to compare the new vacuum surface with the earlier one to ensure that the modification was not at the expense of higher interface pressures. It should be noted that the vacuum stretcher is the support surface of choice in other European countries and that it is mainly only Britain and the USA that use the spinal board.

We were also interested to evaluate and compare other surfaces in common use in the transportation of casualties, and were pleased to be approached to evaluate a new prototype combining vacuum stretcher and spinal board.

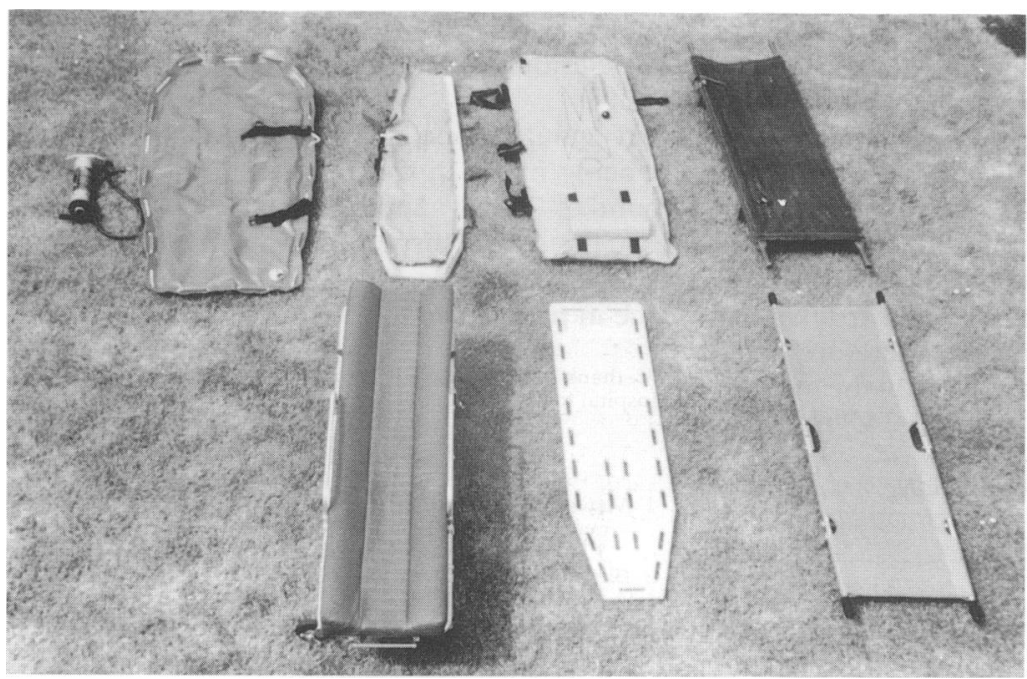

Fig 2 Support surfaces tested: top row left to right: old vacuum stretcher, prototype board, new vacuum stretcher, army stretcher. Bottom row: York Two, spinal board, PVC stretcher.
Often a vacuum stretcher or spinal board is not available and casualties are transported on conventional surfaces. With this in mind we evaluated three other more conventional surfaces. It was also thought that these would give useful baseline information in comparison to the spinal support surfaces.

\section{Methods}

Opportunity to evaluate several support surfaces was available to our team of Territorial Army medics present at the 1994 TT motorcycle races. The method described by Versluysen involving the hand-held Talley pressure meter was used. ${ }^{7}$ An interface pressure pad was applied to the most prominent area of the sacrum and thoracic kyphosis of four members of our team. These subjects then lay on six well established support surfaces and on the prototype support surface. The subjects were arranged so that they were not placed on the surfaces in any particular order and this decreased the risk of bias to the results. The pressure sensor was securely fixed to the subject's skin with adhesive strapping to prevent its movement during transfer between surfaces.

The surfaces evaluated are shown at fig 2 and included a conventional spinal board, a conventional ambulance stretcher with trolley (York 2), the older and larger vacuum stretcher (which allows the casualty to be wrapped and held secure), and a vacuum stretcher of smaller proportions, which more easily fits into conventional vehicles. Our prototype stretcher was a combination of spinal board and vacuum stretcher. We also looked at two conventional stretchers, the Army design compromising a wood and canvas construction and one of aluminium and PVC. These are not considered to be particularly useful in the transportation of the spinal injured but it was thought useful to obtain reference values to compare with the more rigid surfaces. Readings for each surface were taken, and the mean pressures and standard deviations were calculated to measure the variability of sacral and thoracic pressures between the subjects and surfaces. The subjects were asked about general comfort and acceptability of the surfaces. Ease of operation and loading of the surfaces on to vehicles was considered.

\section{Results}

The spinal board had the highest interface pressures at the sacrum and thoracic kyphosis (figs 3 and 4). There was a great deal of variability in the pressures recorded between the subjects and the support surfaces. The least variability was found with the conventional stretchers, including the York Two. The ratio between the thoracic and sacral pressures was between $1: 3$ and $1: 2$. The new vacuum stretcher was found to be the most comfortable surface of the spinal support surfaces, and the ambulance stretcher the most comfortable of the others. The mean values for sacral and thoracic interface pressures are shown at the table. An analysis of variance for both thoracic and sacral pressures showed the distributions 


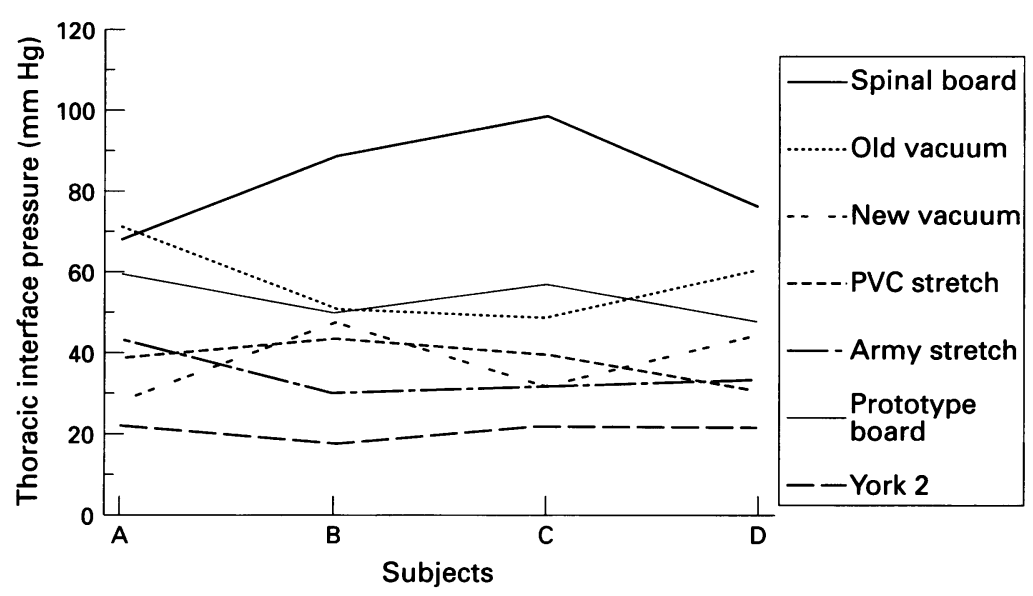

Fig 3 Thoracic interface pressures.

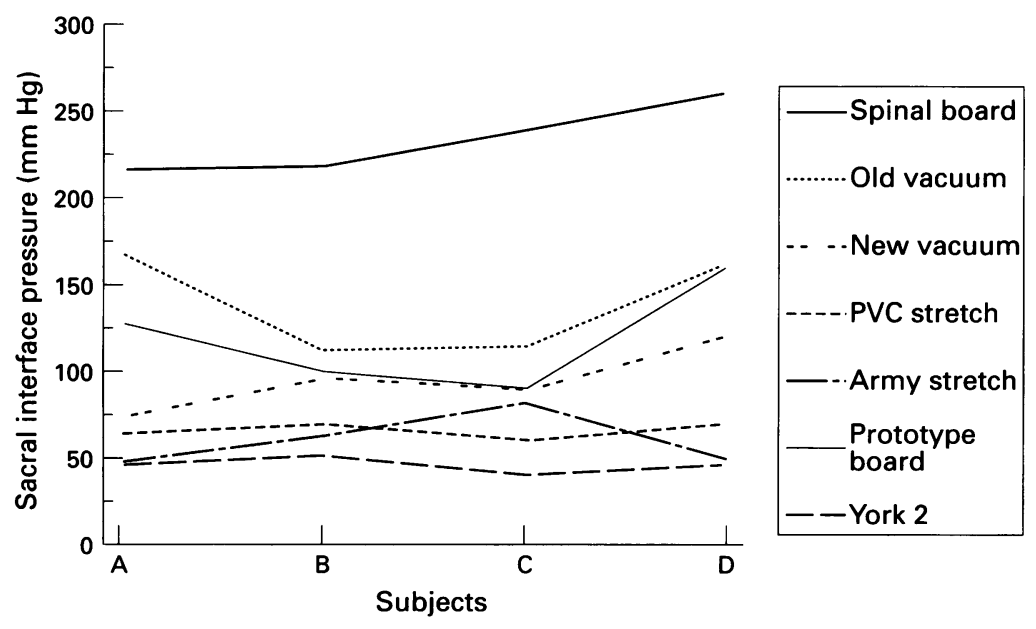

Fig 4 Sacral interface pressures. again a dangerously high value. ${ }^{8}$ We did not have other subjects available for evaluation on all the surfaces, although readings were $c$ available for two subjects on the vacuum $\vec{b}$ surfaces and values less than $100 \mathrm{~mm} \mathrm{Hg}$ ㅇ. were achieved. Further evaluation with more $m$ complex pressure measuring systems would $\underset{\nabla}{\overrightarrow{0}}$ have been useful but were not available to us.

The pressures on the spinal board are $\bar{Z}$ enormous and damaging. Because of the inverse relation between pressure and the rate $\vec{\Rightarrow}$ of tissue necrosis the problem is obviously more than vascular ischaemia and there must be a direct tissue effect. ${ }^{5}$

Other researchers have described problems with the spinal board. Chan found that healthy subjects could experience severe pain lying on $\%$ such a hard surface and this might confuse the $\overrightarrow{0}$ diagnosis or exclusion of actual injury ${ }^{9}$; two other investigators have shown that the cervical $\vec{\omega}$ spine may be put under stress - flexion in $\frac{D}{D}$ young children ${ }^{10}$ and extension in adults. ${ }^{11}$

Overall, the best support surface of those $\vec{\omega}$ used for spinal protection was the new vacuum stretcher, both for interface pressures and $\underset{\perp}{\omega}$ subject comfort. Of the other surfaces, the ambulance stretcher had the best result for comfort and interface pressures, although the $c$ other stretcher surfaces provided reasonable results and would be safe in the short term. Although there is no good evidence about the efficacy of spinal support surfaces it is very difficult, if not impossible, to devise a study to test this, since it would be dangerous to disregard these precautions and patients with suspected injuries require spinal immobilisation. However, patients may be harmed if left on the same hard support surface for a lengthy period. Therefore transportation and investigation of these subjects needs be rapid after initial stabilisation at the scene.

Our prototype hybrid support surface performed well, giving readings better than the old vacuum surface and just slightly less than the new vacuum stretcher. We feel it can be made even better by the addition of more beads and this has been reported to the designer.

Although the old vacuum stretcher and the two conventional stretchers could only be loaded with some difficulty into conventional vehicles, all the other surfaces could be loaded easily.

It would appear that reasonable progress is being made in the evaluation and improvement of spinal support surfaces. We feel that casualties with suspected spinal injuries should be transported on a vacuum type support surface in preference to the spinal board.

We thank the ambulance service at Noble's (Isle of Man) Hospital for use of their equipment and premises. to be less, but in the previous study the average pressure on the spinal board was $147 \mathrm{~mm} \mathrm{Hg}$,

Mean sacral and thoracic pressures $(\mathrm{mm} \mathrm{Hg})$

\begin{tabular}{llc}
\hline & Thoracic pressure & Sacral pressure \\
\hline Spinal board & $82 \cdot 9$ & 233.5 \\
Old vacuum & 58 & 139 \\
New vacuum & $37 \cdot 8$ & $94 \cdot 8$ \\
Prototype & $53 \cdot 7$ & $119 \cdot 5$ \\
York Two & 21 & 46 \\
Army stretcher & $35 \cdot 4$ & 61 \\
PVC stretcher & $38 \cdot 5$ & 66 \\
\hline
\end{tabular}

1 Mawson AR, Biundo JJ, Neville P, et al. Risk factors for early occurring pressure ulcers following spinal cord injury. $A m$ occurring pressure ulcers following

2 Bliss MR. Prevention and management of pressure sores. Update 1988;36:2258-68.

DHSS Working Group. Pressure sores, a key quality indicator. $A$ guide for NHS purchasers and providers. London: Department of Health, 1993.

4 Daniel RK, Priest DL, Wheatley DC. Etiologic factors in pressure sores: an experimental model. Arch Phys Med
Rehabil 1981;62:492. 
5 Reuler JB, Cooney TG. The pressure sore: pathophysiology and principles of management. Ann Intern Med 1981; 94:661-6.

6 Bader DL, Gant CA. Changes in transcutaneous oxygen tension as a result of prolonged pressures at the sacrum Clin Physics Physiol Meas 1988;9:33-40.

7 Versluysen M. How elderly patients with femoral fracture develop pressure sores in hospital. $B M 7$ 1986;292:1311-3.

8 Lovell ME, Evans JH. A comparison of the spinal board and the vacuum stretcher, spinal stability and interface pressure. Injury 1994;25:179-80.
9 Chan D, Goldberg R, Tascone A, Harmon S, Chan L. The effect of spinal immobilization on healthy volunteers. Ann effect of spinal immobilization

Emerg Med 1994;23:48-51.
10 Herzenberg JE, Hensinger RN, Dedrick MD, Philips WA Michigan AA. Emergency transport and positioning of young children who have an injury of the cervical spine. f Bone foint Surg (Am) 1989;71:15-22.

11 Schriger DL, Larmon B, LeGassick T, Blinman T. Spinal immobilization on a flat back board: does it result in neutral position of the cervical spine? Ann Emerg Med $1991 ; 20: 878-8$.

\section{ADVANCED LIFE SUPPORT GROUP}

Major Incident Medical Management and Support Courses: to be held in various centres throughout the UK in 1996. This is a three day course in "life support style" designed to train health service personnel to provide an effective response at a major incident.

Fee $£ 300.00$

Advanced Paediatric Life Support Courses: to be held in various centres throughout the UK in 1996. This is a three day course designed to provide training which will enable doctors and nurses to deal efficiently with all paediatric emergencies. The course is modular and has sections on paediatric resuscitation, serious illness and serious injury.

Fee varies according to centre. Range $£ 300.00-£ 350.00$.

Inquiries for both courses to:

Jenny Antrobus

Advanced Life Support Group

A\&E Dept

Hope Hospital

Stott Lane

Salford M6 8HD

Tel 01617874345 